\title{
La utilización de pilares transmucosos definitivos de colocación inmediata
}

\section{The use of immediate placement of definitive transmucosal abutments}

\author{
A. Isabel Nicolás-Silvente*, A. Rivas-Pérez**, R. García-López**, \\ J. Alemán-Marín**, F. Chiva-García***, A. Sánchez-Pérez****
}

\section{RESUMEN}

El uso de pilares de cicatrización transmucosos previos a la colocación del,pilar protésico definitivo es una técnica que se ha desarrollado durante muchos años en la implantología clásica. La desconexión y conexión de forma repetida de estos pilares o aditamentos dan lugar a una respuesta ósea negativa que se manifiesta en forma de pérdida de hueso a nivel de la cresta marginal, acompañada por una migración apical de tejidos blandos. Este artículo pretende realizar una revisión de la literatura del estado actual del uso de los pilares transmucosos definitivos con colocación inmediata el día de la inserción de los implantes y el mantenimiento del sellado biológico, minimizando la pérdida ósea periimplantaria y remodelando de forma adecuada los tejidos blandos adyacentes, frente al protocolo tradicional del uso de aditamentos que se desconectan en multiples ocasiones produciendo pérdida ósea periimplantaria. La evidencia científica sugiere que los mejores resultados biológicos, estéticos y funcionales se consiguen con el uso del pilar definitivo colocado de forma inmediata a la inserción del implante, especialmente en implantes colocados de forma yuxtacrestal y en implantes postextracción.

PALABRAS CLAVE: Pilar transepiteliar inmediato, ancho biológico, carga tardía, implantes dentales.

\section{ABSTRACT}

The use of transmucosal healing abutments before the placement of the definitive prosthetic abutment is a technique that has been developed for many years in classical implantology. Repeated disconnection and connection of theses abutments result in a negative bone response that manifests as bone loss at the marginal ridge level, accompanied by apical soft tissue migration. This article aims to review the current status in the scientific literature of the use of the definitive transepithelial abutments with immediate placement on the day of implant insertion and maintenance of the biological seal, minimizing peri-implant bone loss and adequately remodeling the adjacent soft tissues, compared to the traditional protocol of the use of abutments that are disconnected on multiple occasions producing peri-implant bone loss. Scientific evidence suggests that the best biological, aesthetic, and functional results are achieved with the use of the definitive abutment placed immediately after implant insertion, especially in yuxtacrestal implants and post-extraction implants.

KEY WORDS: Immediate transepithelial abutment, biological width, delayed loading, dental implants.

\footnotetext{
* Profesor Asociado Dpto. Patología y Terapéutica Dental. Profesor del Máster en Cirugía Mucogingival, Periodontal e Implantológica. Facultad de Medicina. Universidad de Murcia.

** Profesor Colaborador Dpto. Patología y Terapéutica Dental. Facultad de Medicina. Universidad de Murcia.

*** Profesor Titular Dpto. Patología y Terapéutica Dental. Facultad de Medicina. Universidad de Murcia.

**** Profesor Contratado Doctor Dpto. Periodoncia. Facultad de Medicina. Universidad de Murcia.
} 
Fecha de recepción: 14 de abril de 2020

Fecha de aceptación: 22 de abril de 2020

A. Isabel Nicolás-Silvente, A. Rivas-Pérez, R. García-López, J. Alemán-Marín, F. Chiva-García, A. Sánchez-Pérez. La utilización de pilares transmucosos definitivos de colocación inmediata. 2020; 36, (2): 99-106.

\section{INTRODUCCIÓN}

La terapia mediante implantes dentales es una excelente opción de tratamiento para pacientes parcial o totalmente desdentados. Para obtener los resultados óptimos con esta terapia, es preciso que se desarrolle de forma adecuada el proceso de osteointegración, es decir, una unión entre la superficie del implante y el hueso del huésped sin tejido interpuesto. Sin embargo, no solo es necesario que el implante se integre, sino que además es necesaria una relación óptima entre la conexión del implante y el pilar protésico y un buen sellado biológico, el cual, en la mayoría de los casos, se ve alterado en numerosas ocasiones por la conexión y desconexión de diferentes pilares durante el proceso de restauración del implante ${ }^{(1)}$.

\section{Características de la Mucosa}

Perilmplantaria. Sellado Biológico.

La mucosa periimplantaria presenta ciertas similitudes, paro también algunas diferencias con el tejido periodontal, y resulta relevante estudiar histológicamente dichas diferencias. En primer lugar, si analizamos los tejidos blandos que rodean al diente natural, nos encontramos con tres tipos de epitelio: epitelio oral, epitelio de unión y epitelio del surco. Estas estructuras de fijación al diente actúan de protección para evitar la entrada de productos exógenos ya que la cavidad oral está expuesta continuamente a ataques físicos, químicos y bacterianos. Es lo que se denomina anchura biológica y suele tener aproximadamente $3 \mathrm{~mm}$. Por otro lado, cuando se coloca un implante se crean también tres tipos de epitelio: epitelio oral, epitelio periimplantario y epitelio periimplantario del surco. La mucosa alrededor del implante también crea un sellado comparable al del diente natural, sin embargo, la anchura biológica se ve aumentada y es de 3 a $4 \mathrm{~mm}$ aproximadamente. Gould et al. (2) demostraron que las células epiteliales se unen a la superficie del titanio de forma similar a como se une el epitelio a la superficie dental.

A pesar de que el epitelio está en contacto con el pilar y el cuerpo del implante, la continua reabsorción de hueso alrededor del pilar del implante hace que la unión del epitelio sea sólo a nivel del cuerpo del implante. Aunque el epitelio que rodea al implante tiene una estructura muy similar al que rodea al diente natural, su capacidad de sellado es mucho menor, ya que falta la barrera natural que forman el epitelio de unión y el ligamento periodontal ${ }^{(1)}$. Como consecuencia, se producen frecuentemente rece-

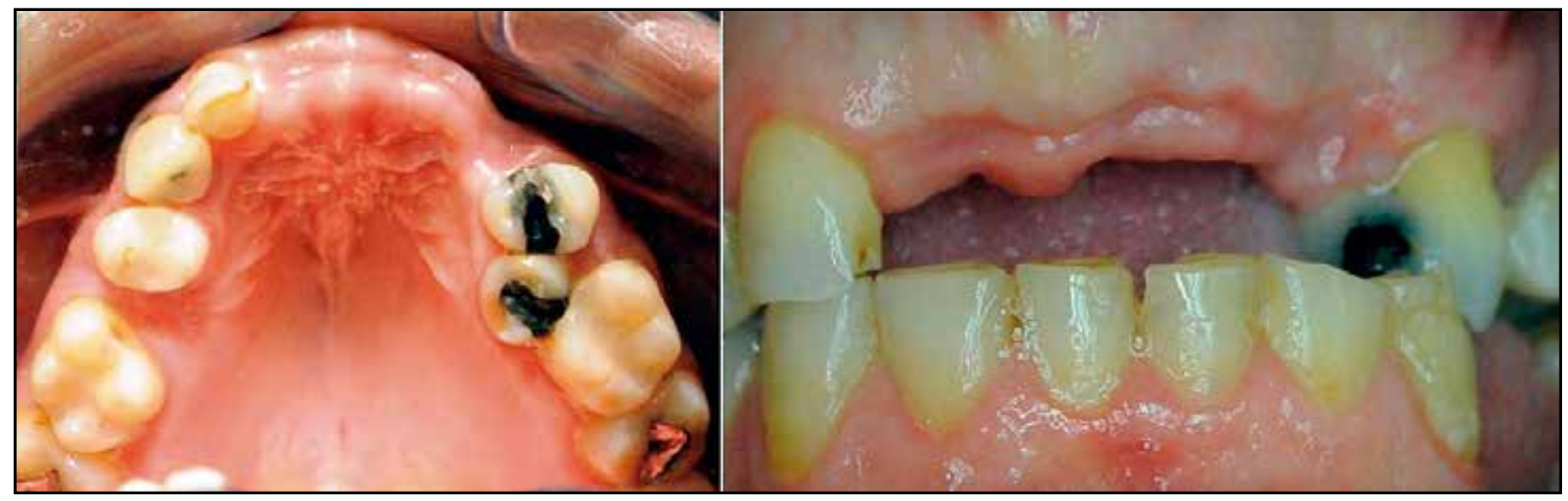

Figura 1. Aspecto preoperatorio oclusal y de frente de maxilar superior parcialmente edéntulo. 4 semanas postextracción. 


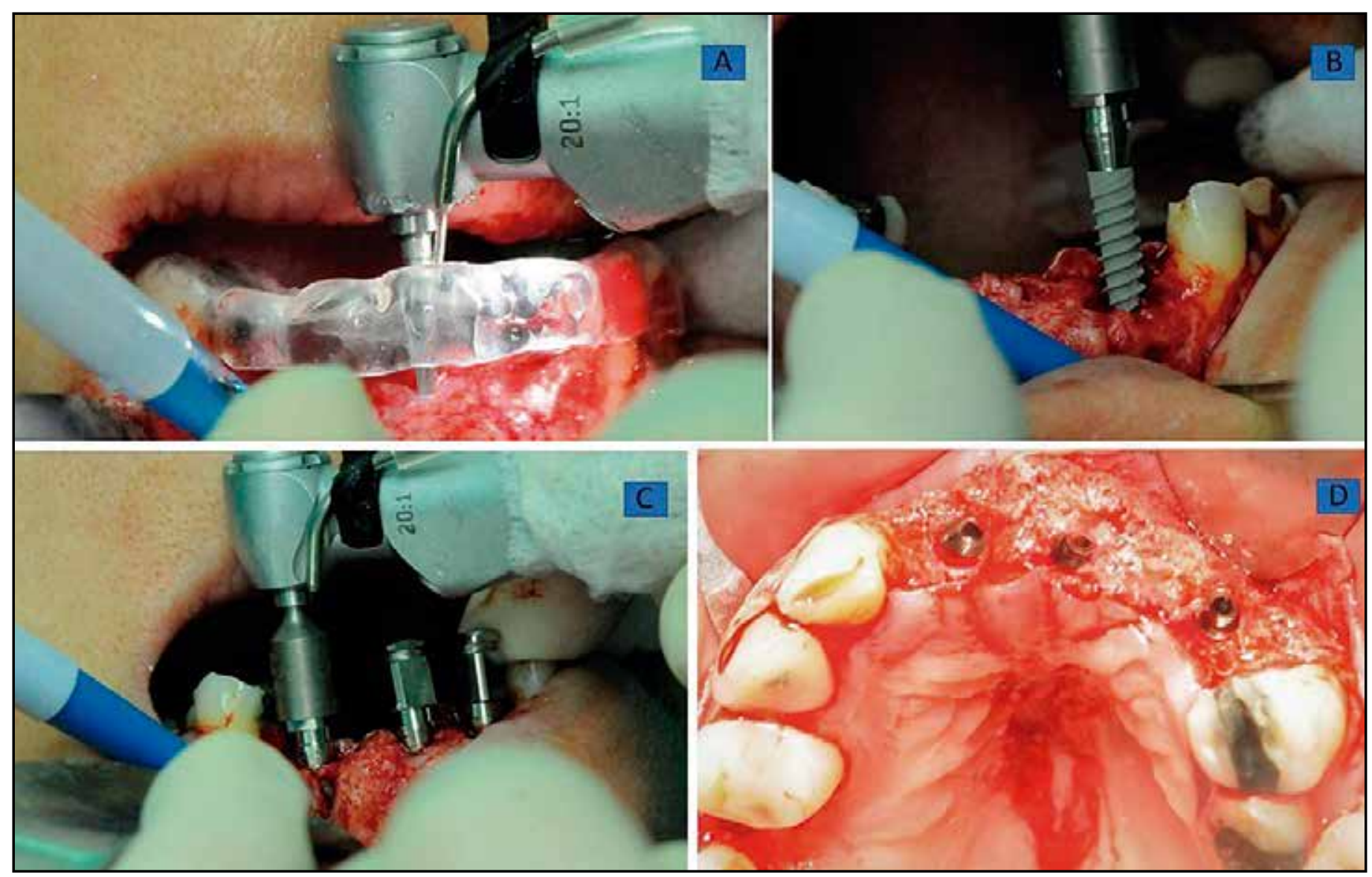

Figura 2. Imagen clínica de cirugía de inserción de implantes. (A) Fresado del lecho implantario con fresa de inicio a través de férula de cirugía inicialmente guiada. (B) Inserción de implante Galimplant IPX. (C) Inserción de implante de forma paralela. (D) imagen oclusal con posición subcrestal de los implantes.

siones 6 meses después de colocar implantes lo que compromete de forma significativa la estética, sobre todo si se trata del sector dentario anterior; y también conduce al fracaso del tratamiento a largo plazo ${ }^{(3)}$.

Al colocar un implante, los tejidos blandos que lo rodean crean una barrera en forma de primera fijación a la superficie del implante, que bloquea el movimiento y la entrada de bacterias $y$ toxinas orales en el espacio entre el implante y los tejidos biológicos, sellando la región. Cualquier daño en el epitelio de unión puede provocar la pérdida de la capacidad de protección y la pérdida ósea continua alrededor del implante ${ }^{(4)}$.

Para mejorar este sellado biológico, tan importante para el éxito a largo plazo del implante, algunos autores han sugerido la modificación de la superficie del implante con proteínas o péptidos que tienen capacidad de unión celular, con el fin de aumentar la adhesión a las células gingivales ${ }^{(5)}$.
El epitelio tiene una rápida proliferación, creciendo sobre la superficie de la fibrina que se deposita de forma inmediata sobre el aditamento protésico. Una vez contactado con el aditamento, se desplaza en sentido corono-apical, originando el epitelio de unión de unos $2 \mathrm{~mm}$ aproximadamente, el cual se une a la superficie metálica mediante hemidesmosomas en los primeros dos o tres dias tras la cirugía. Tanto la presencia de tejido de granulación como la existencia de tejido conectivo inhiben la migración apical ${ }^{(6,7)}$. A diferencia del epitelio adherido a la superficie dental, donde las fibras dentogingivales se dirigen de forma perpendicular/oblicua hacia la superficie radicular y se anclan en el cemento radicular mineralizado, en la superficie del implante, debido a la ausencia de cemento, se produce una adaptación del tejido conectivo, donde las fibras de colágeno se disponen de forma paralela a la superficie del pilar/implante y se orientan en sentido corono-apical o circunferencial ${ }^{(8-10)}$. 

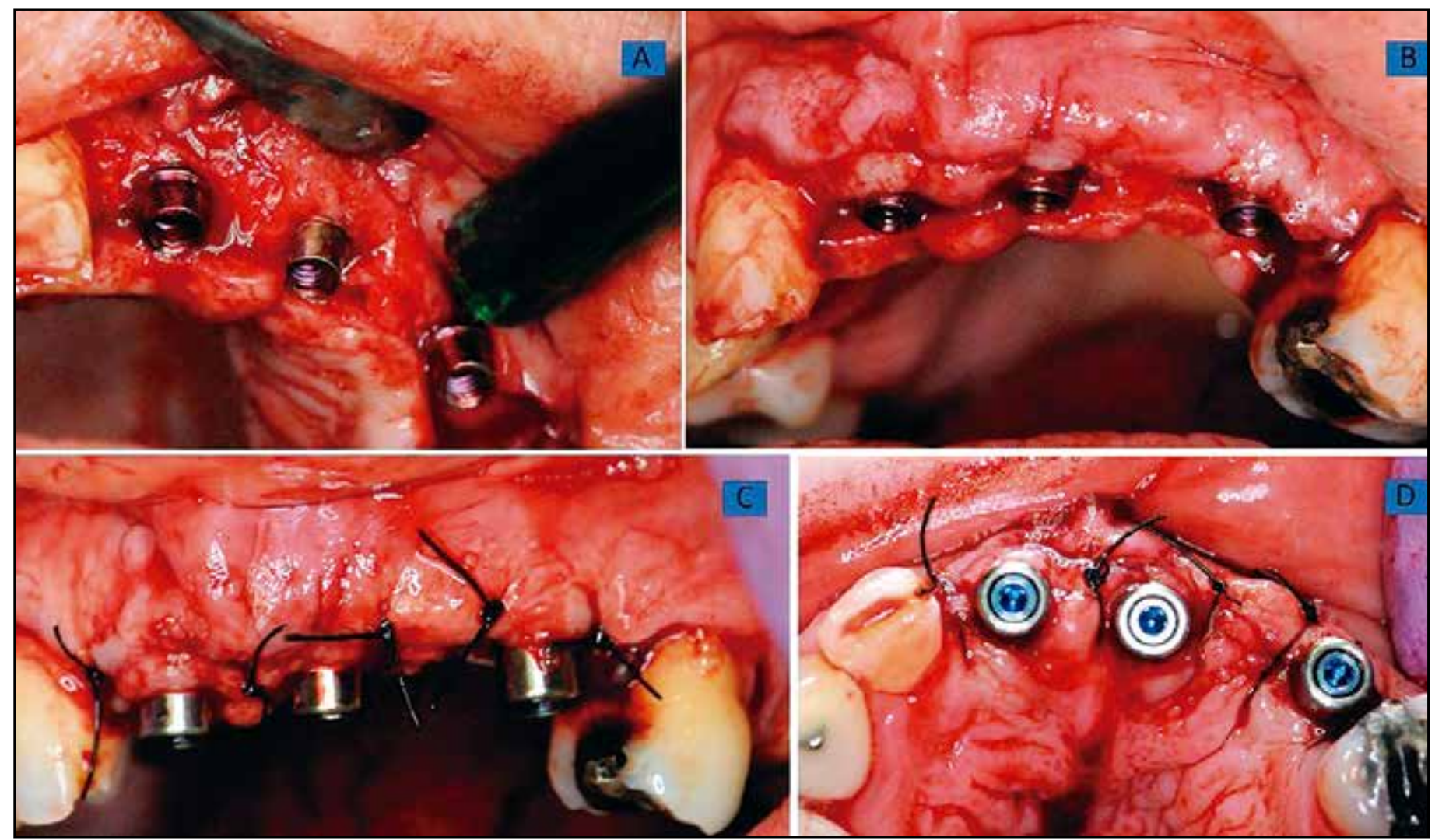

Figura 3. Imagen clínica del pilar transmucoso colocado de forma simultánea. (A) Ajuste del hombro del pilar al reborde óseo marginal. (B) Adaptación del tejido blando perimplantario sin suturar. (C) Visión frontal del pilar transmucoso con las tapas de cicatrización atornilladas. (D) Visión oclusal del pilar transmucoso con las tapas de cicatrización atornilladas.

RELACión de LA INTERFASE IMPLANTE-

Pilar (GAP) con el Tejido PeriImplantario.

Existen dos grandes tipos de implantes, los monofásicos, en los que existe una única interfase (implante-prótesis) y que generalmente se colocan a nivel de encía (tissue level); y los implantes bifásicos, en los que nos encontramos dos interfases (implante-pilar; y pilar-prótesis), y en función de dónde se coloque la plataforma del implante (supra-, yuxta- o sub-crestal) nos dejarán un micro-gap más o menos cercano a la zona crítica, que es el margen óseo. La posible invasión bacteriana del micro-gap podría ser la responsable de la reabsorción ósea tras la producción de infiltrado inflamatorio a ese nivel ${ }^{(11)}$. En los implantes colocados de forma supracrestal, el componente transmucoso donde se forma el espacio biológico es parte del cuerpo del implante y no contiene ningún micro-gap a ese nivel.

Algunos autores han demostrado que existe una mayor migración apical de tejidos blandos y una mayor reabsorción ósea con el uso de implantes yuxtacrestales, sugiriendo que la posición del micro-gap es de vital importancia en la remodelación tisular ${ }^{(12)}$.

Las diferencias de diseño en las conexiones implante-pilar pueden influir en el riesgo de colonización bacteriana. En este sentido, se puede suponer que el papel de la conexión pilar - implante, con respecto al ajuste preciso entre los componentes y la estabilidad mecánica, es de considerable importancia para el éxito terapéutico a largo plazo. La búsqueda de nuevos diseños con el objetivo de minimizar la presencia de ese microespacio y sus efectos, llevó a la introducción de la conexión cono morse como una alternativa prometedora. Los sistemas de conexión externa eran las conexiones más comunes y geométricamente se caracterizaban por la presencia de un hexágono externo. Este diseño tiene muchas desventajas, como la poca superficie de contacto entre la restauración y la parte hexagonal de la cabeza del implante. Existe un cierto grado de rotación entre la plataforma y el hexágono interno de la restauración, generando una gran tensión en la cone- 

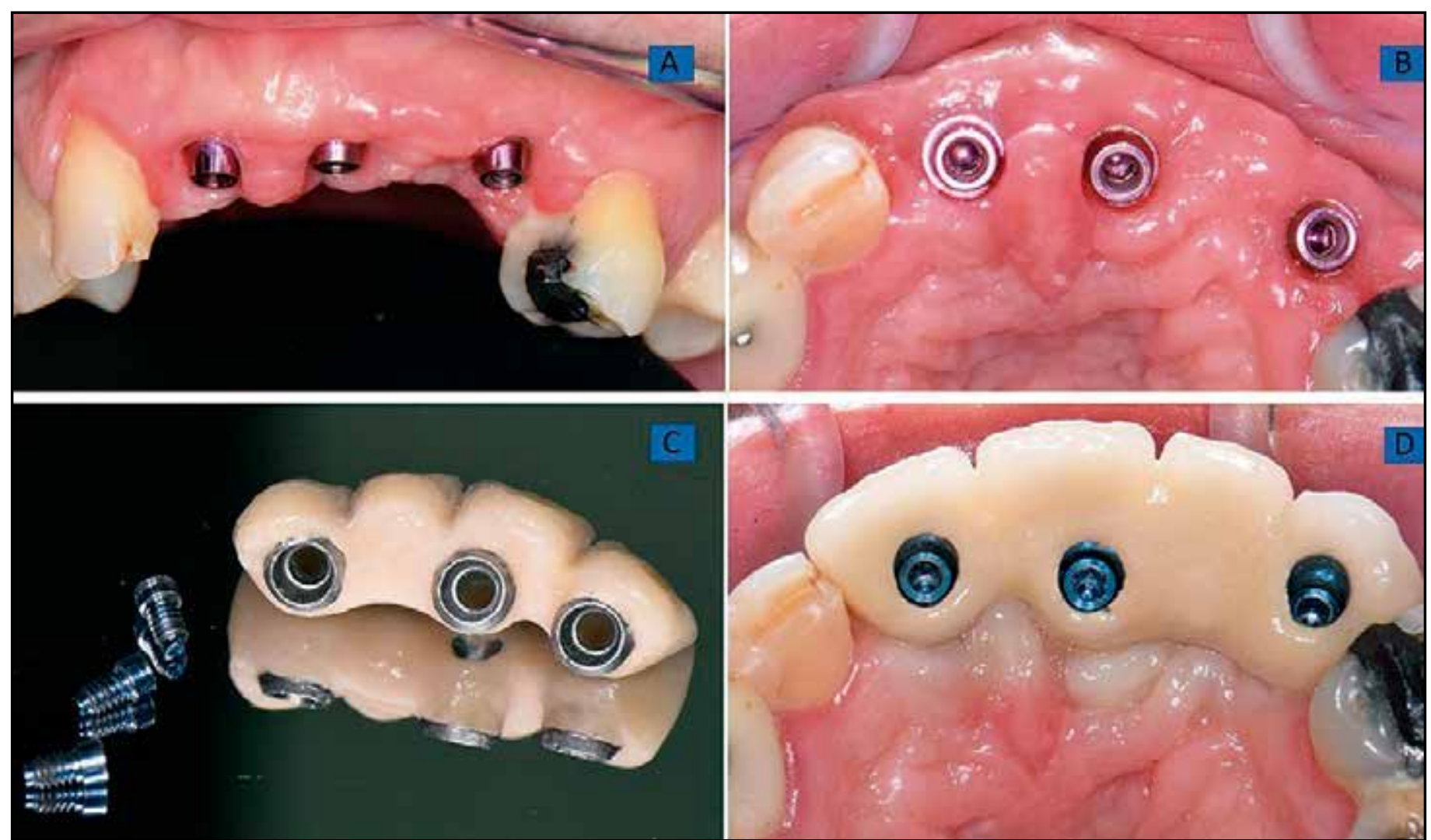

Figura 4. Imagen clínica 3 meses postquirúrgica. (A) Visión frontal y (B) Visión oclusal del aspecto del tejido blando periimplantario. (C) Detalle del puente fijo atornillado sobre los pilares multiposición estéticos. (D) Prótesis recién atornillada donde se puede apreciar la adaptación y la isquemia producida sobre el tejido blando.

xión a nivel del tornillo protésico. Posteriormente, con el sistema hexagonal interno, el hexágono y el tornillo pasaban al interior del cuerpo del implante para que el componente protésico fuera más estable. La fuerza generada en este tipo de conexión se disipa en las paredes adyacentes al hexágono del implante.

La conexión cono morse posee un diseño geométrico en el que un cono con una angulación entre 2 y 4 grados se incrusta en otro cono por fricción, sin la necesidad de tornillos, creando una soldadura en frío entre las dos partes. Hay estudios que demuestran que este tipo de conexión presenta niveles más bajos de microfiltración frente a otro tipo de conexiones ${ }^{(13,14)}$ y, además, el grado de microfiltración bacteriana disminuye según aumenta el torque ${ }^{(15)}$.

Otra estrategia para disminuir la colonización bacteriana y la consiguiente pérdida de hueso y periimplantitis es la introducción del concepto de cambio de plataforma, cuya eficacia viene avalada por diversos estudios recogidos por una re- ciente revisión sistemática y metaanálisis realizado por Wang et al. ${ }^{(16)}$. Este concepto está basado en el uso de pilares con un diámetro menor en comparación con el diámetro de la plataforma del implante, creando así una falta de coincidencia entre ambos componentes a nivel de la interfase implante-pilar. La interfase queda más distante del punto de contacto hueso-implante. Esta configuración da como resultado el aumento en la superficie del implante horizontal que puede alejar el infiltrado inflamatorio del tejido conectivo de la cresta ósea marginal. El cambio de plataforma podría mantener el hueso cerca de la plataforma del implante, minimizando la pérdida ósea marginal periimplantaria.

\section{Importancia del Protocolo Quirúrgico-Protésico.}

Hay distintas técnicas quirúrgicas de abordaje en la colocación de un implante dental, pudiéndose resumir esquemáticamente en cirugía mínimamente invasiva sin colgajo o cirugía con colgajo. Cuando se procede a la realización de un colgajo, la formación y maduración de los tejidos periim- 
plantarios requiere entre 6 y 8 semanas, en una cirugía sin colgajo, la vascularización es mayor y suele haber menor inflamación y una reepitelización más temprana ${ }^{(17)}$. (Figuras 1-5).

Una desconexión frecuente de los componentes protésicos, especialmente en el periodo de formación y maduración del tejido periimplantario en esas primeras 8 semanas producen un empeoramiento en las condiciones de dicho tejido. Puleo y cols. ${ }^{(18,19)}$ establecieron una correlación a nivel de cambios histológicos entre la ruptura mecánica de la interfase periimplantaria y la pérdida de tejido óseo y de tejidos blandos. La repetida desconexión del pilar genera la ruptura de células en el epitelio y a nivel del tejido conectivo, que favorece la migración apical de las células del epitelio de unión, estabilizándo-

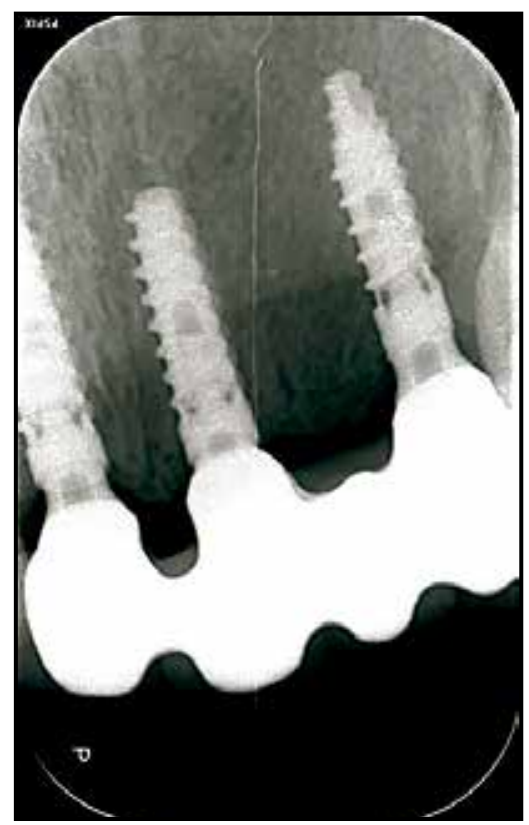

Figura 5. Imagen de radiografia periapical donde se puede observar el hueso de la cresta alveolar que sobrepasa la plataforma del implante y la perfecta adaptación de los componentes protésicos. pilares que se conectan y desconectan sucesivas veces no es significativa hasta la segunda vez y es mayor a mayor número de desconexiones ${ }^{(28)}$.

Con este fin han surgido en el mercado los pilares estéticos multiposición, con un nuevo diseño que buscan conseguir un buen sellado biológico y conseguir un tejido periimplantario adecuado, el cual influye directamente en el resultado estético. Estos pilares están diseñados para conservar la adhesión del tejido blando y mantener una completa flexibilidad restauradora y quirúrgica. Permanece en su posición desde la colocación del implante, durante todo el procedimiento restaurador y durante toda la vida útil de la restauración, manteniendo el tejido blando periimplantario intacto para lograr una cicatrización óptima. Con este se en el primer material inmóvil que encuentren (cuello del implante o pilar), generando una bolsa de tejido blando inestable, que tenderá a producir recesión, especialmente en pacientes con biotipo fino o moderado ${ }^{(20,21)}$.

Abrahamsson et al. (22) demostró que la reabsorción ósea marginal se veía aumentada entre 0.78 y $1.49 \mathrm{~mm}$. tras cinco desconexiones y reconexiones del pilar y observó que esta pérdida ósea marginal se veía asociada a un reposicionamiento apical de los tejidos blandos. Estos resultados se vieron confirmados por los estudios realizados posteriormente por otros autores como Canullo et al. ${ }^{(23)}$ que observaron una menor pérdida ósea marginal en implantes con pilar definitivo inmediato frente a implantes que recibieron un pilar provisional que fue desconectado y conectado en sucesivas ocasiones en implantes colocados en alveolos postextracción.

Diversos autores han demostrado mediante estudios clínicos que se obtienen mejores resultados cuando se coloca el pilar definitivo en el.momento de la inserción del implante ${ }^{(24,25)}$, especialmente en implantes postextraccion ${ }^{(26,27)}$. procedimiento se simplifica mucho el tratamiento restaurador ya que estos pilares trasladan la plataforma restauradora de los implantes de conexión cónica del nivel óseo al nivel tisular, facilitando en gran medida la manipulación de los componentes protésicos y la toma de impresión, ya que la conexión queda visible.

\section{CONCLUSIONES.}

La evidencia científica sugiere mejores resultados a nivel de reabsorción ósea periimplantaria cuando se ven disminuidas las desconexiones y reconexiones del pilar, consiguiendo mejores resultados biológicos, estéticos y funcionales en aquellos casos donde se coloca el pilar de forma inmediata en el mismo momento de la cirugía de inserción de implante y no se vuelve a desconectar, manteniendo integro el sellado biológico en todo momento. Este hecho es de especial relevancia en implantes colocados de forma yuxtacrestal y en implantes postextracción.

\section{BIBLIOGRAFIA}

1. Atsuta I, Ayukawa Y, Kondo R, Oshiro W, Mat- 
suura Y, Furuhashi A, Tsukiyama Y, Koyano K. Soft tissue sealing around dental implants based on histological interpretation. J Prosthodont Res. 2016 Jan;60(1):3-11.

2. Gould TR, Brunette DM, Westbury L. The attachment mechanism of epithelial cells to titanium in vitro. J Periodontal Res. 1981;16: 611-616.

3. Chien HH, Schroering RL, Prasad HS, Tatakis DN. Effects of a new implant abutment design on peri-implant soft tissues. J Oral Implantol. 2014 Oct;40(5):581-8.

4. Alves DC, Carvalho PS, Martinez EF. In vitro microbiological analysis of bacterial seal at the implant-abutment interface using two morse taper implant models. Braz Dent J. 2014 JanFeb;25(1):48-53.

5. Marín-Pareja N, Salvagni E, Guillem-Marti J, Aparicio C, Ginebra MP. Collagen-functionalised titanium surfaces for biological sealing of dental implants: effect of immobilisation process on fibroblasts response. Colloids Surf B Biointerfaces. 2014 Oct 1;122:601-610.

6. Lee DW, Kim JG, Kim MK, Ansari S, Moshaverinia A, Choi SH, Ryu JJ. Effect of laser-dimpled titanium surfaces on attachment of epithelial-like cells and fibroblasts. J Adv Prosthodont. 2015;7:138-45.

7. Fukano Y, Usui ML, Underwood RA, Isenhath S, Marshall AJ, Hauch KD, Ratner BD, Olerud JE, Fleckman P. Epidermal and dermal integra-tion into sphere-templated porous poly (2-hydroxyethyl methacrylate) implants in mice. J Biomed Mater Res A. 2010;94:117286.

8. Benic GI, Scherrer D, Sancho-Puchades M, Thoma DS, Hämmerle CH. Spectrophotometric and visual evaluation of peri-implant soft tissue color. Clin Oral Implants Res. 2017;28:192-200.

9. Fuchigami K, Munakata M, Kitazume T, Tachikawa N, Kasugai S, Kuroda S. A diversity of peri-implant mucosal thickness by site. Clin Oral Implants Res. 2017;28:171-176.
10. Kim BS, Kim YK, Yun PY, Yi YJ, Lee HJ, Kim SG, Son JS. Evaluation of peri-implant tissue response according to the presence of keratinized mucosa. Oral Surg Oral Med Oral Pathol Oral Radiol Endod. 2009;107:24-28.

11. Judgar R, Giro G, Zenobio E, Coelho PG, Feres $M$, Rodrigues JA. Biological width around oneand two-piece implants retrieved from human jaws. Biomed Res Int. 2014;2014:850120.

12. Pellicer-Chover $\mathrm{H}$, Peñarrocha-Diago $\mathrm{M}, \mathrm{Pe}-$ ñarrocha-Oltra D, Gomar-Vercher S, Agustín-Panadero R, Peñarrocha-Diago $M$. Impact of crestal and subcrestal implant placement in peri-implant bone: A prospective comparative study. Med Oral Patol Oral Cir Bucal. 2016;21:103-110.

13. Pessoa RS, Sousa RM, Pereira LM, Neves FD, Bezerra FJ, Jaecques SV et al. Bone Remodeling Around Implants with External Hexagon and Morse-Taper Connections: A Randomized, Controlled, Split-Mouth, Clinical Trial. Clin Implant Dent Relat Res. 2017 Feb;19(1):97-110.

14. Gardel NC, Elizabeth FM, Aluísio MO, Daiane CP, Júlio CJ, Marcelo HN. Microbiological Sealing Analysis of a Tapered Connection and External Hexagon System. Int J Dent. 2017; 2017: 3849085 .

15. Larrucea VC, Jaramillo NC, Acevedo AA, San Martín CL. Microleakage of the prosthetic abutment/implant interface with internal and external connection: in vitro study. Clin Oral Implants Res. 2014 Sep;25(9):1078-83.

16. Wang $Q Q$, Ruoxi Di, Chris YC, Hui F, Min H, Quan-Li L. One-time versus repeated abutment connection for platform-switched implant: A systematic review and meta-analysis. PLoS One. 2017; 12(10): e0186385.

17. Lee HJ, Lee J, Lee JT, Hong JS, Lim BS, Park HJ, Kim YK, Kim TI. Mi-crogrooves on titanium surface affect peri-implant cell adhesion and soft tissue sealing; an in vitro and in vivo study. J Periodontal Implant Sci. 2015;45:1206.

18. Puleo DA, Nanci A. Understanding and controlling the bone-imp|ant interface. Biomate- 
rials. 1999;20:2311-2321.

19. Puleo DA, Thomas MV. Implant surfaces. Dent Clin North Am. 2006;50:323-338.

20. Pollanen MT, Salonen Jl, Uitto VJ. Structure and function of the tooth-epithelial interface in health and disease. Periodontol 2000. 2003;31:12-31.

21. Persson LG, Lekholm U, Leonhardt A, Dahlen $\mathrm{G}$, Lindhe J. Bacterial colonization of internal surfaces of Brånemark system implant components. Clin Oral Implants Res. 1996;7:90-95.

22. Abrahamsson I, Berglundh T, Lindhe J. The mucosal barrier following abutment dis/reconnection. An experimental study in dogs. J Clin Periodontol. 1997; 24:568-57.

23. Canullo L, Bignozzi I, Cocchetto R, Cristalli MP, Iannello G. Immediate positioning of a definitive abutment versus repeated abutment replacements in post-extractive implants: 3-year follow-up of a randomised multicentre clinical trial. Eur J Oral Implantol. 2010;3:285-296.

24. Dorkhan M, Yücel-Lindberg T, Hall J, Svensäter G, Davies JR. Adherence of human oral keratinocytes and gingival fibroblasts to na- no-structured titanium surfaces. BMC Oral Health. 2014; 14:75.

25. Valente NA, Andreana S. Peri-implant disease: what we know and what we need to know. J Periodontal Implant Sci. 2016; 46:136-51.

26. Buser D, Weber HP, Donath K, Fiorellini JP, Paquette DW, Williams RC. Soft tissue reactions to non-submerged unloaded titanium implants in beagle dogs. J Periodontol. 1992;63:225235.

27. Stanford CM. Surface modifications of dental implants. Aust Dent J. 2008;53(suppl 1):S26-S33.

28. Rompen E. The impact of the type and configuration of abutments and their (repeated) removal on the attachment level and marginal bone. Eur J Oral Implantol. 2012;5 Suppl:S83-90

\section{AUTOR DE CORRESPONDENCIA:}

Ana Isabel Nicolas-Silvente

Clinica Odontológica Universitaria.

Hospital Morales Meseguer, $2^{\mathrm{a}}$ Planta.

Avda. Marqués de los Vélez, S/N. 30008 Murcia

Email: ainicolas@um.es 